\title{
Quantitative Relationship between Readiness and Availability of Weapon System and Its Application
}

\author{
Jianwei Lv, Zongren Xie* and Yifan Xu \\ Dept of Management Science \\ Naval Univ. of Engineering \\ Wuhan 430033, China \\ *Corresponding author
}

\begin{abstract}
The definition and calculation of the operational readiness and availability of weapon system have been described. The relationship and difference between them have been analyzed. On the assumption that the repair time, logistic delay time and the relaxation time are subject to different distributions, the models and reasoning of combinations of different distribution and modeling ideas were carried out, the reasonability and typical numerical calculations have been presented according to the modeling results and input data. The trade-offs analyses between the reliability, maintainability and testability indicators based on the modeling results are briefly discussed, and several enlightening conclusions are obtained.
\end{abstract}

Keywords-weapon system; readiness rate; availability rate; quantitative relationship; application

\section{INTRODUCTION}

Operational readiness and availability of weapon system are two concepts that have been used for many years, closely relate to each other and have similar connotations. However, in specification \& standard, academic book and paper, there have not been clear conclusions in their connections and differences, including their modeling and quantitative calculation between them[1-3]. This leads to a lot of confusions and inconvenience in practical engineering application. This paper focuses on engineering application issues in the development of navy vessel and its weapon system and carries out the quantitative modeling analysis and exploration in this aspect.

\section{DEFINITION AND CALCULATION OF READINESS RATE}

Readiness rate of weapon system $P_{O R}$ is an indicator to operational readiness. It means, at any moment, system capability to start the execution of preset mission under peace time and combat conditions. According to this definition and relevant analyses [4], the calculation of readiness rate should take into account the operation, repair and support of system. For instance, a system can still execute the present combat task when there is not failure requiring repair during execution of mission, or any failure requiring repair happens, but its repair time is shorter than the permissible relaxing time of the task. Hence, readiness rate $P_{O R}$ can be calculated by using the following equation.

$$
P_{O R}=R(t)+Q(t) \times P\left(t_{m}<t_{d}\right)
$$

In which, $R(t)$ is the probability of no failure when weapon system executes the previous task, $Q(t)$ is the probability of failure to the previous task, $t$ is the duration of the previous task (h), $t_{m}$ is the time of failure repair, $t_{d}$ is the duration from emerge of failure to the start of next task (h) or the repair time (relaxation time) permitted by the next task [5].

According to this definition, operational readiness can be also presented as follows:

$$
P_{O R}=R(t)+(1-R(t)) \times P\left(t_{m}<t_{d}\right)
$$

Clearly, it is difficult to quantitatively measure the readiness rate of weapon system at the stage of research and development. Due to the uncertainness of mission time $t$, failure repair time $t_{m}$ and relaxation time $t_{d}[6,7]$, it is normally believed that readiness rate of weapon system can be actually measured under the practical combat conditions after executing the assigned mission and task.

\section{DEFINITION AND CALCUlation OF AvailabiLity RATE}

Availability refers to the degree of system being at the ready to work when it is needed to start the execution of task at any time. The probability measure of availability is availability rate. To be specific, it is the ability to put weapon system into operation at any time. In terms of operation time of weapon system, availability can be classified into intrinsic availability $A_{i}$ and operational availability $A_{O}$. The expression for $A_{0}$ is as follows:

$$
A_{0}=\frac{M T B F}{M T B F+M T T R+M L D T}
$$

In which, $M T B F$ is mean time between failure, which is a reliability design parameter of weapon system, MTTR is mean time to repair, which is a maintainability design parameter of weapon system, and $M L D T$ is mean logistic delay time, which is a measure parameter for support ability.

Intrinsic availability only takes into account the availability of corrective repair time. In terms of form, it is the availability indicator that overlooks mean logistic delay time, so there is: 


$$
A_{i}=\frac{M T B F}{M T B F+M T T R}
$$

Since the availability indicator of weapon system comprehensively reflects design parameters including reliability, maintainability and supportability, it is often regarded as a supportability integration parameter. This indicator is also closely related to multiple design parameters of weapon system, so it is often used as a substitute indicator of operational readiness parameter at research and development stage.

\section{PRELIMINARY ANALYSIS ON RELATIONSHIP BETWEEN READINESS RATE AND AVAILABILITY RATE}

When Equation (2) is used for a weapon system, $R(t)$ can be approximately described the percentage of system available time in total task time within the period of the previous task, while $P\left(t_{m}<t_{d}\right)$ is the probability when failure repair time is lower than relaxation time. Thus, Equation (2) is revised to:

$$
P_{O R}=A_{0}+\left(1-A_{0}\right) \times P\left(t_{m}<t_{d}\right)
$$

This equation expresses approximately the relationship between readiness rate and availability rate. In other words, when relaxation time $t_{d}=0$, there is $P\left(t_{m}<t_{d}\right)=0, P_{O R}=A_{O}$. Therefore, a reference pointed out ${ }^{[7]}$ that availability is the representation of operational readiness to specific weapon system. With regard to their difference, availability is judged based on whether weapon system can function or not. If system is in repair, it is not available and all failure repair time is entirely unavailable. Operational readiness is judged based on the success of mission. When system is at the state of failure, but repair time does not exceed relaxation time, it will not affect the execution of mission, so weapon system is considered ready to work, and calculation of readiness rate is not affected. The relaxation time permitted by mission is a random factor determined by specific combat mission, force structure, composition and combat need to weapon system, support ability, etc. If relaxation time is $0, P_{O R}$ and $A_{O}$ have the same value. If relaxation time is higher than $0, P_{O R}$ must have a higher value than $A_{O}$, but it is still incomparable to $A_{i}$. In other words, the analysis based on Equation (5) does not solve the problem of quantitative relationship for readiness rate and availability rate.

\section{MODELING FOR RELATIONSHIP BETWEEN READINESS RATE AND AVAILABILITY RATE}

Since there is seldom basic research on this problem, it is necessary to explore the modeling principle and distribution of random variables involved in modeling.

With regard to variables involved in modeling, it can be first assumed that failure repair time $t_{m}$, relaxation time $t_{d}$ and delay time $t_{s}$ are all random variables. Among them, $t_{m}$ and $t_{s}$ are subject to exponential distribution $[8,9]$ (whose mean values are MTTR and MLDT respectively), while $t_{d}$ can be arranged to follow exponential distribution (whose mean value is $M R T$ ) or normal distribution [10].

During modeling, the above variables are handled as follows:

(1) Under normal circumstances, if $t_{d}>t_{m}+t_{s}$, there is $P_{O R}={ }_{A}$; if not, there is $P_{O R}=A_{O}$.

(2) When $t_{m}<<t_{s}$, if $t_{d}>t_{s}$, there is $P_{O R}=A_{i}$; if not, there is $P_{O R}=A_{O}$.

(3) Equation (5) is used as model to calculate.

Obviously, the last two cases belong to the approximation for the convenience of calculation. By the distribution of the above variables and the idea to process, 6 cases of combinations about distribution and modeling idea can be obtained as follows:

No.1: $t_{m}, t_{s}$ and $t_{d}$ are all subject to exponential distribution.

No.2: $t_{m}$ and $t_{s}$ are subject to exponential distribution, $t_{d}$ subject to normal distribution.

No.3: $t_{m}<<t_{s}$ can be ignored, $t_{s}$ and $t_{d}$ are subject to exponential distribution.

No.4: $t_{m}<<t_{s}$ can be ignored, $t_{s}$ is subject to exponential distribution, $t_{d}$ is subject to normal distribution.

No.5: Calculation using Equation (5), so it involves only $t_{m}$ and $t_{d}$, all subject to exponential distribution.

No.6: Calculation using Equation (5), so it involves only $t_{m}$, subject to exponential distribution, and $t_{d}$, subject to normal distribution.

Among these 6 cases, No.1, 3 and 5 can have analytic solution and obtain clear calculation results, while other cases cannot have analytic solution. Due to limited space and for the convenience of expression, only the illustrating process for case No.1 is presented and the others are omitted.

In case No.1, to a specific repair activity, by the above analysis and assumption, we have:

$$
f(z)=f\left(t_{m}+t_{s}\right)=f\left(t_{m}\right) * f\left(t_{s}\right)=\int_{0}^{z} \mu e^{-\mu(z-z)} l e^{-t y} d y=\frac{\mu l}{l-\mu}\left(e^{-\mu}-e^{-k}\right)
$$

In which, $\mu=1 / M T T R, \quad l=1 / M L D T$. Meanwhile, there is $\lambda_{d}=1 / M R T$.

When $t_{d}>t_{m}+t_{s}$, there is:

$$
P\left\{t_{d}>t_{m}+t_{s}\right\}=\frac{l \cdot \mu}{(l-\mu)} \int_{0}^{+\infty}\left(e^{-\mu}-e^{-k}\right) d z \int_{=}^{+\infty} \lambda_{d} e^{-\lambda_{d} t} d t=\frac{l \cdot \mu}{\left(\mu+\lambda_{d}\right)\left(l+\lambda_{d}\right)}
$$


When $t_{d}<t_{m}+t_{s}$, there is:

$$
P\left\{t_{d}<t_{m}+t_{s}\right\}=\frac{l \cdot \mu}{(l-\mu)} \int_{0}^{+\infty}\left(e^{-\mu \varepsilon}-e^{-k z}\right) d z \int_{0}^{z} \lambda_{d} e^{-\lambda_{d} t} d t=\frac{\lambda_{d}\left(\mu+l+\lambda_{d}\right)}{\left(\mu+\lambda_{d}\right)\left(l+\lambda_{d}\right)}
$$

From Equations (7) and (8), there is:

$$
P_{\text {oR }}=\frac{l \cdot \mu}{\left(\mu+\lambda_{d}\right)\left(l+\lambda_{d}\right)} \times A_{i}+\frac{\lambda_{d}\left(\mu+l+\lambda_{d}\right)}{\left(\mu+\lambda_{d}\right)\left(l+\lambda_{d}\right)} \times A_{o}
$$

All the results of modeling are presented in TABLE I.

The above modeling and compute results can be preliminarily analyzed as follows:

(1) For the evolution from No.1 to No.3, we can see, if $t_{m}<<t_{s}$ can be ignored, there is $\mu>>\lambda_{d}, \therefore \mu+\lambda_{d} \approx \mu$, $\mu+l+\lambda_{d} \approx \mu$. Hence, No.1 in Equation (9) is evolved into the No.3. In other words, case No.3 is approximate to No.1 under special circumstances.

\begin{tabular}{|c|c|c|c|c|}
\hline $\begin{array}{l}\text { Case } \\
\text { No. }\end{array}$ & Distribution Type and Handling Method & $\begin{array}{l}\text { Analytic } \\
\text { Solution } \\
\end{array}$ & $A_{i}$ 's Coefficient & $A_{O}$ 's Coefficient \\
\hline 1 & $t_{m}, t_{s}$ and $t_{d}$ (exponential). & Yes & $\frac{l \cdot \mu}{\left(\mu+\lambda_{d}\right)\left(l+\lambda_{d}\right)}$ & $\frac{\lambda_{d}\left(\mu+l+\lambda_{d}\right)}{\left(\mu+\lambda_{d}\right)\left(l+\lambda_{d}\right)}$ \\
\hline 2 & $t_{m}$ and $t_{s}$ (exponential), $t_{d}$ (normal). & No & $\int_{0}^{+\infty} \frac{1}{\sigma \sqrt{2 \pi}}\left(\frac{l e^{-\mu x}-\mu \mathbf{e}^{-l x}}{l-\mu}\right) e^{\frac{(x-\mu)^{2}}{2 \sigma^{2}}} d x$ & $\int_{0}^{+\infty} \frac{1}{\sigma \sqrt{2 \pi}} \frac{l e^{-\mu x}-\mu \mathrm{e}^{-l x}}{l-\mu} e^{\frac{\left(x-\mu_{0}\right)^{2}}{2 \sigma^{2}}} d x$ \\
\hline 3 & $\begin{array}{c}t_{m}<<t_{s} \text { ignored, } \\
t_{s} \text { and } t_{d} \text { (exponential). }\end{array}$ & Yes & $\frac{l}{\lambda_{d}+l}$ & $\frac{\lambda_{d}}{\lambda_{d}+l}$ \\
\hline 4 & $\begin{array}{c}t_{m}<<t_{s} \text { ignored, } t_{s} \text { (exponential) } \\
t_{d} \text { (normal). }\end{array}$ & No & $\int_{0}^{+\infty} \frac{1}{\sigma \sqrt{2 \pi}}\left(-e^{-l x}\right) e^{-\frac{\left(x-\mu_{0}\right)^{2}}{2 \sigma^{2}}} d x$ & $\int_{0}^{+\infty} \frac{1}{\sigma \sqrt{2 \pi}} e^{-l x} e^{-\frac{\left(x-\mu_{0}\right)^{2}}{2 \sigma^{2}}} d x$ \\
\hline 5 & $\begin{array}{c}\text { Calculation using Equation (5), involving } \\
\text { only } t_{m} \text { (exponential) and } t_{d} \\
\text { (exponential) }\end{array}$ & Yes & \multicolumn{2}{|c|}{$P_{O R}=A_{O}+\left(1-A_{O}\right) \times \frac{\mu}{\mu+\lambda_{d}}$} \\
\hline 6 & $\begin{array}{l}\text { Calculation using Equation (5), involving } \\
\text { only } t_{m} \text { (exponential) and } t_{d} \text { (normal). }\end{array}$ & No & \multicolumn{2}{|c|}{$P_{O R}=A_{O}+\left(1-A_{O}\right) \times \int_{0}^{+\infty} \frac{1}{\sigma \sqrt{2 \pi}}\left(1-e^{-\mu x}\right) e^{-\frac{\lambda x}{2 \sigma^{2}}} d x$} \\
\hline
\end{tabular}

TABLE I . MODELING RESULTS FOR CASE NO.1-NO.6

$1 / 3$ shipment-level repair with delay of 0

Personnel
Spare Parts

$90 \%$ spare parts sufficiency, 0

$10 \%$ delay, $36 \mathrm{~h}$

$1 / 3$ depot-level repair with delay of 24

\section{FIGURE I. COMBINATIONS OF PERSONNEL DELAY AND SPARE PARTS DELAY}

(2) Considering the MLDT's current level and the future development trend, the calculation results of No.5 have poor accuracy.

(3) If $t_{d}$ employs normal distribution, it will result in troublesome integral approximate calculation on the interval $[0$, $+\infty$ ], and there are evidences indicating that the assumption of normal distribution is not reasonable to some extent. The situation of battlefield is changing all the time, and weapon system must execute various missions. The order assigning time of next task and its relaxation time can't vary near a central time range. At present, the limited statistical results (here omitted) tend to support this judgment. Hence, the further analysis on case No.2, 4 and 6 is abandoned.

(4) Based on the current research level and data, it is recommended to use the equation of case No.1 for calculation.

\section{SAMPLE ILLUSTRATION AND THE RESULT'S APPLICATION}

\section{A. Calculation Results of Typical Sample}

In the stage of weapon system's research and development, $M T B F$ and MTTR have clear definitions and been widely studied and applied. As an example ,some typical data are given herein, that is, : $M T B F=1000, M T T R=2$. Similarly, we can take $M R T=12 \mathrm{~h}$.

It must be noted that there is not universal method for calculation and analysis of $M L D T$ indicator ${ }^{[11-13]}$. Here we give the following typical calculation approach and results for navy vessel's repair based collected data. 
For ship's repair activities, MLDT consists of personnel delay, spare parts delay and administration delay. The three parts are analyzed and calculated as follows:

(1)Personnel Delay: Assuming that equipment's failure onboard has $1 / 3$ shipment-level, intermediate-level and depotlevel recovered respectively (resource data omitted) and their personnel delays are as same. And personnel delay for shipment-level repair is 0; personnel delay for intermediate level repair is $1-3 \mathrm{~h}$, taking the average value of $2 \mathrm{~h}$; and personnel delay for depot-level repair is $24 \mathrm{~h}$.

(2) Spare Parts Delay: Considering the above three types of repair, the sufficiency of spare parts is $90 \%$. In other words, there is no spare parts delay (sufficient spare parts onboard) at the rate of $90 \%$. The remaining $10 \%$ spare parts delay takes $36 \mathrm{~h}$ according to the practice of U.S. navy.

Two kinds of delays above are presented in FIGURE I.

As revealed in the above diagram, the combination time of such two delays is $12.30 \mathrm{~h}$.

(3) Administration Delay: Administration delay generally happens during equipment failure onboard, and its time can be 2-3h. Thus, $M L D T=f$ (personnel delay, spare parts delay, administration delay) $=15 \mathrm{~h}$.

By the typical data above, $A_{i}=0.998004$ and $A_{o}=0.982994$ can be get. Then $P_{O R}$ 's calculation result can be get in TABLE II.

TABLE II. CALCULATION RESULTS OF $P_{O R}$

\begin{tabular}{|c|c|c|c|c|}
\hline $\begin{array}{c}\text { Combina } \\
\text { tion No. }\end{array}$ & $\begin{array}{c}\text { Analytic } \\
\text { Solution }\end{array}$ & $\begin{array}{c}A_{i} \text { 's } \\
\text { Coefficient }\end{array}$ & $\begin{array}{c}A_{O} \text { 's } \\
\text { Coefficient }\end{array}$ & $P_{O R}$ \\
\hline 1 & Yes & 0.376766091 & 0.623233909 & 0.988649381 \\
\hline 3 & Yes & 0.43956044 & 0.56043956 & 0.989591911 \\
\hline 5 & Yes & - & - & 0.9975706 \\
\hline
\end{tabular}

Based on the result, the input data are changed one by one to analyze the sensitivity of parameters and verify the conclusion of the previous analysis. For instance, let $M T T R=0.3$, the calculation results of case No.3 and No.1 are near same, so calculation results verify the analysis conclusion follow TABLE 1 . Let $M L D T=3$, the calculation results of case No.5 are obviously abnormal, reflecting that approximate results of Equation (5) have poor accuracy in some combination of parameters.

\section{B. Application in Trade-off among Reliability, Maintainabi- lity and Testability Indicators}

The above analysis results can be applied widely. For instance, Equation (2) can be revised to:

$$
P_{O R}=R(t)+P_{D} \times(1-R(t)) \times M\left(t_{d}\right)
$$

In which, $P_{D}$ is fault detection rate, which is one of major testability indicators of equipment, and $M\left(t_{d}\right)$ is maintain degree within relaxation time $t_{d}$. Under the support of other parameters, this model can be used to calculate the required fault detection rates at system or equipment level. If this equation is combined with other models, such as, Markov chain model [14] and analogue method, etc., it can be used to determine the required maintainability and testability indicators at system or equipment level based on the existing reliability of equipment in a concurrent way. Also, it can effectively eliminate the current situation that maintainability and testability indicators of equipment are subjectively determined without theoretical basis, and improve that RMT (Reliability, Maintainability, Testability) indicators have no interdependence and cannot be cooperated among them, so as to provide better theoretical basis for equipment's RMT design and development of weapon system.

\section{CONCLUSION}

Operational readiness and availability are two important concepts in the research \& development and operation of weapon system. The systematic modeling analysis on their relationship can help us identify the quantitative relationship between readiness rate and availability rate on the basis of existing research, and can be also used in the trade-off analysis of reliability, maintainability and testability indicators at the research \& development stage of weapon system. This paper presents the definition of such two concepts and their calculation methods, and conducts the modeling analysis and quantitative calculation based on their relationship and difference, so as to guide practical engineering. The built models and calculation results have been applied in the quantitative analysis on a ship's system-level testability indicators. The application has revealed that the calculation results based the model presented in this paper can satisfy the requirements of engineering application, and results fulfill the expected goal.

\section{ACKNOWLEDGMENT}

This work was supported by National Natural Science Foundation of China (71401171), PLA General Armament Department Pre-research Fund (9140A19030214JB11273), Military Universities 2110 Projects Phase III (4142D4557).

\section{REFERENCES}

[1] Kobren, Bill. Product Support and Human Capital: Essential Ingredients for Optimizing System Readiness, Availability, and Life Cycle Costs[R].Defense Acquisition Univ., FT Belvoir VA, 2011,8.

[2] Charles Elegbede, Kondo Adjallah. Availability Allocation to Repairable Systems with Genetic Algorithms: A Multi-Objective Formulation[J]. Reliability Engineering and System Safety, 2003,82: 319-330.

[3] Moongon Jung, Joydeep Mitra, David Z. Pan, Sung Kyu Lim. TSV stress-aware full-chip mechanical reliability analysis and optimization for 3DIC[J]. Communications of the ACM,2014,57(1):107-115.

[4] MAO, Bingxiang, BAI Hua, CHENG Wenxin. System Operational Readiness: Analysis, Computation and Detection [M]. China National Defense Industry Press, 2012.

[5] LV Jianwei, YU Peng, YANG Jianjun. Research on Time Relaxation \& Sailing Policy of Navy Vessel during Seaway for Task [J].Journal of Naval University of Engineering, 2012, 24(2): 25-28.

[6] G. V. Vorobev, K. S. Pshenichnaya. Methodology of the system analysis of the influence of testability indicators on the usage efficiency of 
aircraft weapons systems[J]. Journal of Computer and Systems Sciences International, 2012, 51(5): 663-680.

[7] Mark Saunders, Wayne Richie, John Rogers. Predicting mission success in small satellite missions[J].Acta Astronautica, 2003, 52(2): 381-370.

[8] Koehn P, Macheret Y, Sparrow D. Improving Reliability and Operational Availability of Military Systems [R]. Alexandria: Institute for Defense Analyses, 2004.

[9] Defense AT\&L. Army Logistics Readiness and Sustainability[M]. USA: Policy \&Legislation, 2004.

[10] SUN Lei, JIA Yunxian, WANG Shuai. Research on MLDT modeling method [J]. Computer \& Digital Engineering, 2011,39(1): 21-24.

[11] HOU Na, LI Yi, WANG Yigang, LIU Junjie, ZHANG Bo, LV Xuezhi Research on the Mean Logistic Delay Time of the Development Phrass[J]. Physics Procedia,2012,33:375-379.

[12] XIE Hongcheng, WEI Jia. Research on Calculation Simulation for Multi-level Stores MLDT and Integration Evaluation Model for Determining Spare Parts Variety[C]//2009. IIS'09. International Conference on Industrial and Information Systems, 2009.

[13] LI Dongdong, YU Yongli, ZHANG Liu, DONG Yue, NIE Chenglong. Research on transformation from combat unit aerial defence interval mission to MLDT [J]. Systems Engineering and Electronics, 2010, 32(9): 1907-1910.

[14] Taboada H. A, Espiritu J. F., Coit D. W.. MOMS-GA:A Multi-Objective Multi-State Genetic Algorithm for System Reliability Optimization Design Problems[J].IEEE TRANSACTIONSON RELIABILITY,2008, 57(1): 182-191. 